\title{
Lack of polymyxin resistance among carbapenemase- producing Enterobacteriaceae in a university hospital in China
}

Sir,

We read with interest two recent articles in the present journal focusing on escalation of carbapenamase-producing Enterobacteriaceae in the hospital environment, one from the Zhejing Province, China [1], and another from Northern Italy [2]. Multidrug resistance is increasingly reported in clinical enterobacterial isolates in China. Not only resistance to carbapenems is on the rise, but in addition co-resistance to other clinically-relevant antibiotics such as fluoroquinolones, aminoglycosides and fosfomycin is increasingly observed. Polymyxins are therefore considered as last resort antibiotics for treating infections due to multidrug-resistant Gram negatives.

Recently, plasmid-mediated colistin resistance (MCR-1) was reported in enterobacterial from animals, food and patients from China [3]. Beyond China, the spread of the mcr-1 gene has been now reported worldwide in many enterobacterial species [4]. In addition, one of the major concern is the spread of carbapenem- and polymyxinresistant nosocomial Klebsiella pneumoniae isolates as observed in Italy [5] and more recently in France [6]. High plasmid-mediated $\mathrm{mcr}-1$ carriage rate has been observed in E. coli collected from raw meat $(15 \%)$ and animals (21\%) in China, but not from inpatients (1\%) [3]. Recently, a variant of MCR-1, namely MCR-2, has been reported from Belgium [7].

Taking into account the wide spread MCR-1-producing strains in China and elsewhere, the diversity of genetic structures associated to the mcr-1 gene identified so far, and the diversity of the clonal backgrounds of the strains harboring that gene, it is likely that its spread does not correspond to a recent event in China.

We have performed a retrospective study to evaluate the occurrence of polymyxin resistance and of the
MCR-1 determinant among carbapenemase-producing Enterobacteriaceae isolates, including $112 \mathrm{~K}$. pneumoniae, 15 Escherichia coli, 15 Citrobacter freundii, 12 Enterobacter aerogenes, 3 Klebsiella oxytoca, 2 Citrobacter farmeri, a single Enterobacter cloacae and a single Citrobacter braakii. Those clinical isolates have been recovered during the 2006-2011 period at the Huashan Hospital of Fudan University, Shanghai. Ninety-four were from sputum, 43 from urine, 6 from drainage, 5 from blood, 3 from cerebrospinal fluid and 10 from other samples. Carbapenemase activity was assessed using the Rapidec Carba NP test (bioMérieux, La Balme-les-Grottes, France) [8]. Carbapenemase genes were searched as previously described [7]. All isolates were screened for polymyxin resistance by using the recently-developed Rapid Polymyxin NP test [9]. MICs of colistin and polymyxin B were determined by broth microdilution method as recommended by CLSI [10]. All polymyxin-resistant isolates were screened for the mcr-1 and mcr-2 genes by using PCR with primers mcr-all-F (5'-TATCGCTATGTGCTAAAG$3^{\prime}$ ) and mcr-all-R (5'-TCTTGGTATTTGGCGGTA-3'). All of 161 isolates were tested positive for the Rapidec Carba NP test. Further PCR and sequencing showed that all strains were positive for $b / a_{\mathrm{KPC}-2}$. No polymyxin-resistant isolate was detected using the Rapid Polymyxin NP test, and further determination of MICs of colistin and polymyxin B confirmed that all isolates were susceptible to polymyxins. Accordingly, all isolates tested negative for the $m c r-1$ and $m c r-2$ genes.

This study revealed the absence of polymyxin-resistant isolate in our collection of carbapenemase-producing enterobacterial isolates. Combining with results obtained through other Chinese studies $[4,6,8]$, our data suggest that polymyxin resistance rates may be much lower than expected in Chinese hospitals. However, surveillance of 
polymyxin resistance shall be implemented on a regular basis to monitor its potential emergence in China.

To conclude, polymyxins might still constitute clinically-relevant options for treating infections caused by carbapenemase-producing Enterobacteriaceae in China. However, the use of polymyxins for humans is still not approved in China, considering its significant toxicity.

\section{Acknowledgements}

This work was supported by the National Natural Science Foundation of China (Nos. 81572031) and by the University of Fribourg.

\section{Disclosure statement}

The authors report no conflicts of interest. The authors alone are responsible for the content and writing of this article.

\section{Funding}

This work was supported by the National Natural Science Foundation of China (Nos. 81572031) and by the University of Fribourg.

\section{References}

[1] Sun Y, Li M, Chen L, et al. Prevalence and molecular characterization of carbapenemase-producing gram-negative bacteria from a university hospital in China. Infect Dis (Lond). 2016;48:138-146.

[2] Caselli D, Cesaro S, Fagioli F, et al. Incidence of colonization and bloodstream infection with carbapenem-resistant Enterobacteriaceae in children receiving antineoplastic chemotherapy in Italy. Infect Dis (Lond). 2016;48:152-155.

[3] Liu YY, Wang Y, Walsh TR, et al. Emergence of plasmid mediated colistin resistance mechanism MCR-1 in animals and human beings in China: a microbiological and molecular biological study. Lancet Infect Dis. 2015;16:161-168.

[4] Du H, Chen L, Tang YW, et al. Emergence of the mcr-1 colistin resistance gene in carbapenem-resistant Enterobacteriaceae. Lancet Infect Dis. 2016;16:287-288.

[5] Capone A, Giannella M, Fortini D, et al. High rate of colistin resistance among patients with carbapenem-resistant Klebsiella pneumoniae infection accounts for an excess of mortality. Clin Microbiol Infect. 2013;19:E23-E30.

[6] Jayol A, Poirel L, Dortet L, et al. National survey of colistin resistance among carbapenemase-producing Enterobacteriaceae and outbreak caused by colistin-resistant OXA-48-producing Klebsiella pneumoniae, France, 2014. Euro Surveill. 2016;21.
[7] Xavier BB, Lammens C, Ruhal R, et al. Identification of a novel plasmid-mediated colistin-resistance gene, $\mathrm{mcr}-2$, in Escherichia coli, Belgium, June 2016. Euro Surveill. 2016;21.

[8] Poirel L, Nordmann P. Rapidec Carba NP test for rapid detection of carbapenemase producers. J Clin Microbiol. 2015;53:3003-3008.

[9] Nordmann P, Jayol A, Poirel L. Rapid detection of polymyxin resistance in Enterobacteriaceae. Emerging Infect Dis. 2016;22:1038-1043.

[10] Clinical and Laboratory Standards Institute (CLSI). Methods for dilution of antimicrobial susceptibility tests for bacteria that grow aerobically. Approved standard, 9th ed. CLSI document M07-A10. Wayne: CLSI; 2015.

Xiaofei Jiang

Emerging Antibiotic Resistance Unit, Medical and Molecular Microbiology, Department of Medicine, University of Fribourg,

Fribourg, Switzerland

French INSERM European Unit, University of Fribourg (LEA-IAME),

Fribourg, Switzerland

National Reference Center for Emerging Antibiotic Resistance, Fribourg, Switzerland

Department of Laboratory Medicine, Huashan Hospital, Shanghai Medical College, Fudan University, Shanghai, China

Laurent Poirel

Emerging Antibiotic Resistance Unit, Medical and Molecular Microbiology, Department of Medicine, University of Fribourg, Fribourg, Switzerland

French INSERM European Unit, University of Fribourg (LEA-IAME), Fribourg, Switzerland

National Reference Center for Emerging Antibiotic Resistance, Fribourg, Switzerland

ه laurent.poirel@unifr.ch

Patrice Nordmann

Emerging Antibiotic Resistance Unit, Medical and Molecular Microbiology, Department of Medicine, University of Fribourg,

Fribourg, Switzerland

French INSERM European Unit, University of Fribourg (LEA-IAME),

Fribourg, Switzerland

National Reference Center for Emerging Antibiotic Resistance,

Fribourg, Switzerland

Department of Laboratory Medicine, Huashan Hospital, Shanghai Medical College, Fudan University, Shanghai, China University of Lausanne and University Hospital Center, Lausanne, Switzerland 